\title{
Molecular dynamics in a nematic liquid crystal probed by implanted muons
}

\author{
B. W. Lovett, ${ }^{*}$ S. J. Blundell, J. S. Stießberger, ${ }^{\dagger}$ F. L. Pratt, ${ }^{\ddagger}$ Th. Jestädt, and W. Hayes \\ The Clarendon Laboratory, University of Oxford, Parks Road, Oxford OX1 3PU, United Kingdom \\ S. P. Cottrell \\ ISIS Facility, Rutherford Appleton Laboratory, Chilton, Didcot OX11 OQX, United Kingdom \\ I. D. Reid \\ Paul Scherrer Institute, Villigen CH-5232, Switzerland \\ (Received 22 August 2000; published 11 January 2001)
}

\begin{abstract}
We present the first muon spin rotation/relaxation study of a nematic liquid crystal (5CB). We identify four different positions for muonium addition to this compound by correlating the dominant peaks in the Fourier transform of the muon precession signal obtained in an applied transverse magnetic field. Experiments performed in a longitudinal field are used to probe changes in individual molecular dynamics at the solid-nematic transition. High field avoided level crossing spectroscopy reveals four $\Delta M=0$ transitions which can be fitted using simplified models of collective molecular dynamics appropriate to the solid, nematic, and liquid phases.

DOI: 10.1103/PhysRevB.63.054204

PACS number(s): 61.30.-v, 76.75.+i, 33.15.Vb
\end{abstract}

\section{INTRODUCTION}

The uniaxial nematic $(N)$ mesophase of a liquid crystal is a state of matter which exhibits symmetry properties intermediate between those of a solid $(S)$ and an isotropic liquid (I) ${ }^{1}$ In the $N$ phase, molecules have translational freedom and orientate preferentially along one particular axis, which is defined by a unit vector called the director $\mathbf{n}$. This orientationally ordered phase arises due to packing constraints of the rod-like molecules. ${ }^{2}$ The $N$ phase has been investigated by a variety of experimental techniques and there has been significant theoretical interest in the nature of the molecular dynamics. ${ }^{1}$ Nuclear magnetic resonance (NMR) measurements have elucidated many of its dynamical properties. ${ }^{3}$ The usefulness of the positive muon, an alternative local magnetic probe, has recently been established in dynamical studies of small organic molecules, ${ }^{4}$ but so far this technique has not been applied to liquid crystals.

To demonstrate the feasibility of applying the muon spin rotation/relaxation ( $\mu \mathrm{SR})$ technique ${ }^{5,6}$ to nematics we present the results of measurements carried out on the nematic compound 4'- $n$-pentyl-4-cyanobiphenyl (5CB), whose molecular structure is shown in Fig. 1. It has a $S-N$ transition at $24^{\circ} \mathrm{C}$ and a $N-I$ transition at $35^{\circ} \mathrm{C}$. It is chemically the simplest nematic mesogen known and is thus a good choice for this first investigation.

The paper is divided into five sections. Section II describes the procedure for determination of the muon site. Section III describes the results of experiments performed with a longitudinal magnetic field which we explain by using a model of individual molecular dynamics, not specific to the liquid crystal phase. Section IV discusses avoided level crossing resonance experiments which can be explained by considering director fluctuations in the nematic phase. In Sec. V we present our conclusions and compare our results with NMR.

\section{TRANSVERSE FIELD $\mu$ SR AND MUON SITES}

Transverse field muon spin rotation ( $\mathrm{TF} \mu \mathrm{SR})$ measurements were performed using the general purpose decaychannel spectrometer at the Paul Scherrer Institute (PSI, Switzerland). In these experiments a beam of spin polarized muons was implanted into the sample of $5 \mathrm{CB}$. These muons are polarized longitudinally with respect to the beam direction and the field is applied transverse to this. The time dependence of the decay angles of positrons emitted by the muons is recorded by a series of fast scintillation counters positioned upstream and downstream of the muon beam. Since the decay positrons in this weak decay are emitted preferentially in the opposite direction to the muon spin we can obtain the time evolution of the ensemble muon polarization. ${ }^{4}$

In $5 \mathrm{CB}$, as in other organic materials, we expect muonium $\left(\mathrm{Mu}=\mu^{+} e^{-}\right)$to form by electron capture soon after implantation. $\mathrm{Mu}$ behaves as a light isotope of hydrogen $\left(m_{\mathrm{Mu}}\right.$ $=\frac{1}{9} m_{\mathrm{H}}$ ) and so reacts with unsaturated bonds forming a muonated radical. ${ }^{4}$ We assume throughout this paper that the radical formed has the same dynamical properties as the parent $5 \mathrm{CB}$ because the structure of the muonated $5 \mathrm{CB}$ radical is almost identical to the parent molecule. This approximation is commonly made for muonated radicals of large molecules. ${ }^{7,8}$ The unpaired electronic spin density (S) couples to the muon spin (I) through the isotropic Fermi contact interaction $A_{\mu}$ and the anisotropic magnetic dipoledipole interaction $D_{\mu}$. In an applied magnetic field $\mathbf{B}$, the spin Hamiltonian for such a system is given by

$$
\frac{\mathcal{H}}{\hbar}=A_{\mu} \mathbf{S} \cdot \mathbf{I}+D_{\mu}(\mathbf{S} \cdot \mathbf{I}-3(\mathbf{S} \cdot \hat{\mathbf{u}})(\mathbf{I} \cdot \hat{\mathbf{u}}))-\gamma_{\mu} \mathbf{I} \cdot \mathbf{B}+\gamma_{e} \mathbf{S} \cdot \mathbf{B},
$$

where $\gamma_{e}$ and $\gamma_{\mu}$ represent the gyromagnetic ratios of the electron and muon, respectively, and $\hat{\mathbf{u}}$ is a unit vector along the symmetry axis of the hyperfine tensor. We have assumed 


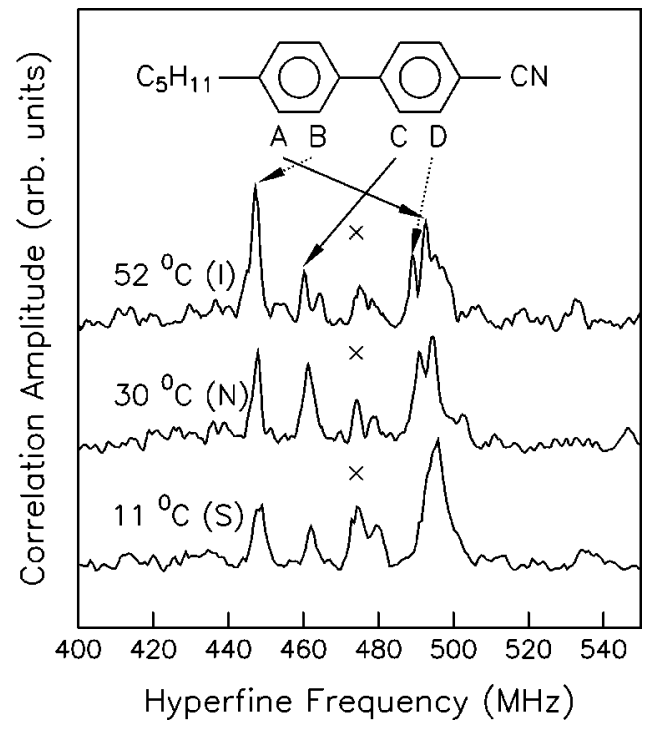

FIG. 1. Structure of 5CB showing four muon positions and corresponding correlation amplitudes obtained from a TF experiment. The cross marks an artifact peak.

uniaxial dipolar coupling, which is valid in the case of fast rotation about one molecular axis as is found in the $N$ phase. The anisotropic dipole-dipole interaction $D_{\mu}$ is effectively zero in the case of fast isotropic tumbling of molecules as would be expected in the $I$ phase.

At high magnetic field $(\gg 20 \mathrm{mT})$ the eigenstates of the muon-electron system are well approximated by the two particle Zeeman states quantized in the field direction, except in the special case of an avoided level crossing (see Ref. 4 and later). Thus if the muons are implanted with their spins transverse to the field, they are in maximally entangled combinations of Zeeman up and down spin states and will thus precess. Two precession frequencies are expected, corresponding to the selection rule $\Delta I_{z}= \pm 1, \Delta S_{z}=0 .{ }^{4}$ These two precession frequencies are obtained by fast Fourier transform of the raw muon time spectra and their average value is $A_{\mu}$.

In a field of $200 \mathrm{mT}$ we identified four pairs of radical precession frequencies (not shown). In a given magnetic field the frequencies of the two peaks in the Fourier spectrum, which are expected for a given value of $A_{\mu}$, can be calculated. It is thus possible to obtain a power spectrum as a function of $A_{\mu}$ by computing the product of the amplitudes of the Fourier strengths at the two frequencies for each possible value of $A_{\mu}$. It is easier to identify the peaks in this correlated spectrum than in the raw Fourier transforms. Four peaks are clearly visible in the correlation amplitude spectrum for $5 \mathrm{CB}$ in the $I$ phase and these then broaden with decreasing temperature as shown in Fig. 1 . In the $I$ phase where $D_{\mu}$ is averaged out, the peaks correspond to four values of $A_{\mu}$. These are due to the four possible inequivalent sites for Mu addition to the 5CB molecule.

It is possible to predict the value of $A_{\mu}$ for each site by using the empirical rules of Roduner. ${ }^{4}$ He finds that for muons added to substituted benzene molecules, $A_{\mu}$ is given by the expression
TABLE I. Calculated and measured values of the muon hyperfine constant for the four inequivalent sites for muon addition to 5CB ( $o=$ ortho and $m=$ meta position).

\begin{tabular}{lcccc}
\hline \hline Site & Substituents used & $\begin{array}{c}A_{\mu}^{\text {calc }} \\
(\mathrm{MHz})\end{array}$ & $\begin{array}{c}A_{\mu}^{\text {meas }} \\
(\mathrm{MHz})\end{array}$ & $\begin{array}{c}A_{n}^{\text {meas }} \\
(\mathrm{MHz})\end{array}$ \\
\hline$A$ & $\mathrm{CH}_{3}(o), \mathrm{C}_{6} \mathrm{H}_{6}(m)$ & 490.4 & $492.70(2)$ & $120.4(1)$ \\
$B$ & $\mathrm{C}_{6} \mathrm{H}_{6}(o), \mathrm{CH}_{3}(m)$ & 459.1 & $447.20(5)$ & $109.0(1)$ \\
$C$ & $\mathrm{C}_{6} \mathrm{H}_{6}(o), \mathrm{CN}^{(m)}$ & 467.1 & $460.3(1)$ & $111.5(1)$ \\
$D$ & $\mathrm{CN}(o), \mathrm{C}_{6} \mathrm{H}_{6}(m)$ & 486.5 & $489.30(5)$ & $121.5(1)$ \\
\hline \hline
\end{tabular}

$$
A_{\mu}=A_{\mu}^{0} \prod_{X}\left(1-\Delta_{X}\right),
$$

where the $X$ represent the substituent groups on the ring and $\Delta_{X}$ is the corresponding empirical shift for that substituent group in one of the three possible inequivalent positions with respect to the muon on the ring (these are called the ortho $[o]$, meta $[m]$, and para $[p]$ positions). In Eq. (2.2), $A_{\mu}^{0}$ is the hyperfine constant for free benzene, $514.6 \mathrm{MHz}$. In order to calculate values for the four muon sites, we have taken the shifts for the measured substituent groups which are chemically the closest to the ones we actually have on our molecules. In Table I we compare the result of the calculation with the assigned sites given in Fig. 1, where it can be seen that the outer muon sites, $A$ and $D$, correspond to the higher frequency peaks and the inner sites, $B$ and $C$, to the lower frequency peaks. The agreement with Roduner's rules is good, and the small discrepancies are probably due to the fact that the the substituent groups used in our calculation are not exactly the same as those that appear in 5CB.

The peak widths are due to instrumental or lifetime broadening and also contain a term that depends on $D_{\mu}$. The increasing widths in the $S$ and $N$ phases give an upper limit for the $D_{\mu}$ of a few $\mathrm{MHz}$ and show that each $D_{\mu}$ decreases with increasing temperature, an effect that is caused by motional narrowing. We will return to a discussion of anisotropy in Sec. IV where we will find accurate values for the $D_{\mu}$ and see that these are crucial in allowing us to determine the dynamic properties of $5 \mathrm{CB}$ using muons.

\section{LONGITUDINAL FIELD MEASUREMENTS AND INTERNAL MOLECULAR DYNAMICS}

Longitudinal field muon-spin relaxation (LF $\mu \mathrm{SR}$ ) measurements were carried out using the EMU spectrometer at the ISIS facility (U.K.). These experiments differ only in geometry from the TF case described in the previous section; the field is applied parallel to the initial direction of the muon spin. This means that the initial state of the muon is now a pure eigenstate at high magnetic field [see Eq. (2.2)] and relaxation in the muon polarization can only occur if there are fluctuations in the hyperfine or dipolar fields at the muon site. This is analogous to a $T_{1}$ process in NMR so that a measurement of the temperature dependence of this relaxation allows us to extract dynamical information concerning 5CB.

We show specimen depolarization curves for the $S, N$, and 


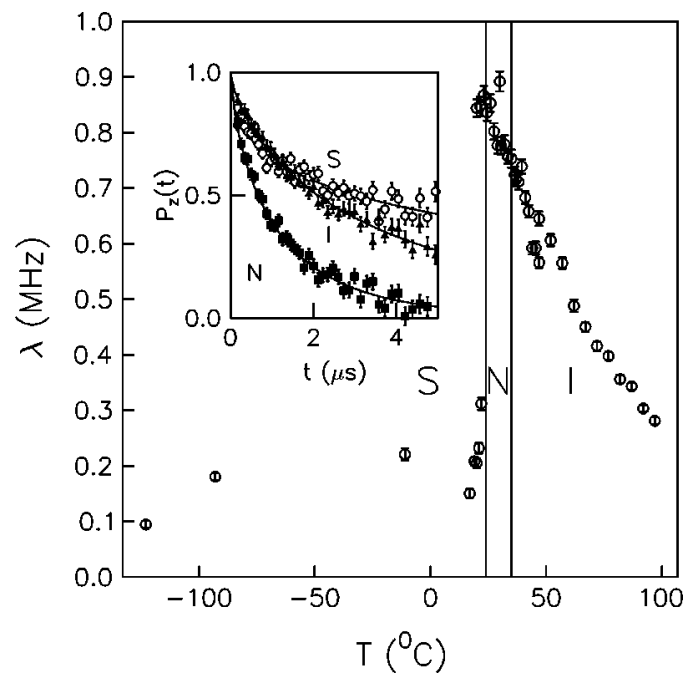

FIG. 2. Main figure: muon relaxation rate as a function of temperature at $50 \mathrm{mT}$. Inset: muon depolarization at $50 \mathrm{mT}$ for the $S, N$, and $I$ phases.

$I$ phases in Fig. 2. We show only data taken in a field of 50 $\mathrm{mT}$ since these demonstrate most clearly the difference between the three phases, providing a compromise between the requirements of a substantial fraction of relaxing polarization (which increases with applied field due to the repolarization of singlet $\mathrm{Mu}$ states) and relaxation rate (which decreases with higher applied field as the $\mathrm{Mu}$ states become more constrained to the external field direction). In order to parametrize the data, we first fit the decaying muon polarization using the phenomenological expression

$$
P_{z}(t)=\exp \left[-(\lambda t)^{\beta}\right]
$$

The temperature dependence of the relaxation rate $\lambda$ is shown in Fig. 2 (we find $\beta \sim 0.8$ across most of the temperature range). The relaxation rate in the $N$ phase is clearly much greater than that in the $S$ phase, a fact that may be attributed to a greater fluctuation rate of the hyperfine interaction between the muon and the electronic spin density. The relaxation rate becomes smaller again at higher temperatures, indicating faster fluctuations and stronger motional narrowing effects.

We may model this effect directly to extract molecular fluctuation rates from the muon relaxation rate by using the dynamical models of Cox and Sivia. ${ }^{9}$ The muon depolarization is modeled as follows. First, it is necessary to calculate the four eigenstate energies $E_{i}$ of the dominant static isotropic Hamiltonian of the muon-electron spin system, without including any smaller time varying interactions. In this state, no depolarization is possible, but the introduction of a fluctuating magnetic perturbation gives rise to resonant depolarization. Hence, the second step is the inclusion of a fluctuating field by considering the transition rate equation between the four levels. This may be represented by a $4 \times 4$ matrix, which describes the effect of this fluctuating perturbation. The off diagonal elements represent the transition rate $W_{i j}$ between two unperturbed eigenstates $i$ and $j$ and are given by the well known Bloembergen, Purcell, and Pound expression $^{10,11}$

$$
W_{i j}=M_{i j}^{2} \frac{\nu}{\nu^{2}+\omega_{i j}^{2}},
$$

where $\omega_{i j}$ is the energy difference in frequency between levels $i$ and $j, M_{i j}$ is a matrix element, and $\nu$ is the fluctuation rate. The diagonal elements are simply found by adding the rates from each of the three other levels and taking the negative to ensure detailed balance. Thermal population factors have been omitted since they are not important for the energy scales we are considering. We assume the strong collision model and that fluctuations happen at times distributed according to the probability distribution $\rho(t)=\nu \exp (-\nu t)$.

The matrix element $M_{i j}$ varies depending on relaxation mechanism. In $5 \mathrm{CB}$, there are two likely contributions to $M_{i j}$. The first is a modulation of the contact term $A_{\mu}$, which would occur through intramolecular modes, i.e., changes in internal vibrational or torsional states. However, this mechanism is characterized by its ineffectiveness in zero field. ${ }^{9}$ The fact that we have observed strong relaxation at zero field suggests that another mechanism is present in $5 \mathrm{CB}$. This second mechanism is that of whole molecule reorientation, which modulates the anisotropic dipolar part of the interaction. We assume that this interaction can be represented by an effective field of strength $B_{D}=(3 / 2){ }^{1 / 2} D_{\mu} / \gamma_{\mu}$, the rms field of a magnetic dipole characterized by a coupling constant $D_{\mu}$. The off diagonal matrix elements coupling the four level system are given approximately by the symmetric matrix $^{9}$

$$
M_{i j}^{2}=\left(\gamma_{\mu} B_{D}\right)^{2}\left(\begin{array}{cccc}
- & (c+s)^{2} & 0 & (c-s)^{2} \\
(c+s)^{2} & - & (c+s)^{2} & 0 \\
0 & (c+s)^{2} & - & (c-s)^{2} \\
(c-s)^{2} & 0 & (c-s)^{2} & -
\end{array}\right) .
$$

We have omitted the diagonal elements which have no meaning here. The diagonal terms in Eq. (3.2) are easily found by using the detailed balance procedure outlined above. The number of particles in each of the four states as a function of time is now calculated by finding the eigenvalues $\lambda_{i}$ and eigenvectors $\mathbf{v}_{\mathbf{i}}$ of the relaxation matrix [Eq. (3.2)]. Since the total number of particles in each of the four levels is conserved, one of these eigenvalues is always zero. This gives rise to three exponentially decaying terms and a constant term:

$$
\left(\begin{array}{l}
n_{1}(t) \\
n_{2}(t) \\
n_{3}(t) \\
n_{4}(t)
\end{array}\right)=A \mathbf{v}_{\mathbf{1}} e^{-\lambda_{1} t}+B \mathbf{v}_{2} e^{-\lambda_{2} t}+C \mathbf{v}_{\mathbf{3}} e^{-\lambda_{3} t}+D \mathbf{v}_{\mathbf{4}}
$$

$n_{1}, n_{2}, n_{3}$, and $n_{4}$ represent the time varying probabilities of occupation of the four eigenstates. For simplicity, we assume 


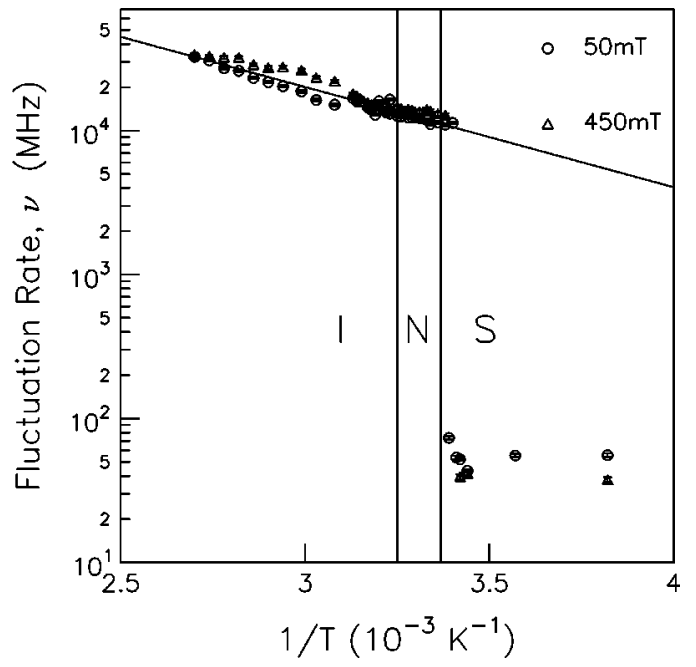

FIG. 3. Molecular fluctuation rate as a function of temperature at 50 and $450 \mathrm{mT}$.

single values for the two hyperfine constants, taking $A_{\mu}$ $=450 \mathrm{MHz}$, a typical value observed in the transverse field experiment, and $B_{D}=2 \pi / \gamma_{\mu} \times 10 \mathrm{MHz}$ (this quantity is explicitly measured in Sec. IV). At $50 \mathrm{mT}$ the four eigenstates are then approximately pure Zeeman states. In a representation having the electron spin direction first and the muon second they are $|1\rangle=|\uparrow \uparrow\rangle,|2\rangle=|\uparrow \downarrow\rangle,|3\rangle=|\downarrow \downarrow\rangle$ and $|4\rangle$ $=|\downarrow \uparrow\rangle$ (in fact levels $|2\rangle$ and $|4\rangle$ admix slightly with each other; this is taken into account but has very little effect on the end result). The muon polarization is now easily found by taking $P_{z}(t)=n_{1}(t)+n_{4}(t)-\left[n_{2}(t)+n_{3}(t)\right]$ and using the constrained initial state of muon up polarization (with half of the electrons in down states and the other half in up states) to determine the prefactors in the above equation. This means that the total polarization is also the sum of three exponentials (in this case the constant term is zero) which, as we determined in our previous fit [Eq. (3.1)], combine in such a way as to give stretched exponential relaxation characterized by $\beta \sim 0.8$.

The temperature dependence of the fluctuation rate $\nu$ for two values of the magnetic field extracted from application of the molecular reorientation model [Eq. (3.4)] is shown in Fig. 3. There is a large increase in its value at the $S-N$ transition as the molecules become free to move. $\nu$ then progressively increases as the temperature is raised, and is well fitted by assuming an activated dependence with an energy barrier $\Delta E=138(6) \mathrm{meV}$. The fitted values at the two fields are in good agreement with one another which suggests that our model is at least a good approximation to the true situation.

The absolute values of $\nu$ and the size of the energy barrier are in reasonable agreement with deuterium NMR results, which have been explained by a combination of internal rotation about the para axis of the phenyl ring within $5 \mathrm{CB}$ and of reorientation of the whole $5 \mathrm{CB}$ molecule. ${ }^{12}$ The relaxing motion is in fact rather complex, being characterized by separate reorientational and internal correlation times in the "superimposed rotations" model of Ref. 12. However, there it was also found that the fluctuation rate follows a simple
Arrhenius law, one possible explanation being that one of the two types of motion has a much larger fluctuation rate and so swamps the other type of motion. This seems to agree with our results; we have used a pure molecular reorientation model, which has worked well for different fields and over a range of temperatures.

The lack of discontinuity in the relaxation rate at the $N-I$ transition seen in our results has been seen before in deuterium $^{12}$ and carbon-13 $\mathrm{NMR}^{13}$ experiments on $5 \mathrm{CB}$, which also find very little change in the activation energy at the $N-I$ transition for probes placed on the benzene ring. This suggests that we are seeing motion not intrinsic to the liquid crystal phase, i.e., that director fluctuations are not important. This would be consistent with a fast rotation of the whole molecule about its long axis, which is not a collective phenomenon.

\section{AVOIDED LEVEL CROSSING SPECTROSCOPY AND COLLECTIVE DYNAMICS}

The avoided level crossing (ALC) technique ${ }^{5}$ uses a similar experimental geometry to that of the LF experiments discussed earlier, but utilizes a larger magnetic field. The time integrated asymmetry $P_{\mathrm{av}}$ is measured as a function of applied magnetic field. For most values of the applied field the Zeeman states are pure eigenstates but at certain fields energy levels anticross. An anticrossing of levels, which mixes up and down muon spin states, gives a longitudinal oscillation of the muon polarization and hence a resonance in the ALC spectrum. It is found that interactions of the radical electron with the muon and with nearby protons are important in determining the positions and shapes of ALC resonances, which can be labeled by the change $\Delta M$ in the sum $M$ of nuclear and muonic spin quantum numbers. Three types of resonance are possible, corresponding to $\Delta M=0,1$, and 2. Kreitzman and Roduner ${ }^{14}$ have developed a theory for the case of a single interacting proton. Their results show that in the case of a static hyperfine tensor the ALC problem is equivalent to a muon precessing in an effective field, which is a combination of the external field and hyperfine interactions. Dynamics may then be introduced by using a Monte Carlo technique ${ }^{15}$ in which the orientation of the symmetry axis of the hyperfine tensor is adjusted according to a particular random probability distribution with an appropriate correlation time. The technique is sensitive to much longer correlation times $(\sim 1 \mu$ s) than those found in Sec. III, because the level splittings are several orders of magnitude smaller. The ALC and LF techniques are thus complementary.

The experiments were carried out on the ALC spectrometer at PSI and typical results are shown in Fig. 4 after background subtraction. We observe four resonances in $5 \mathrm{CB}$. Positions of $\Delta M=1$ transitions can be accurately predicted from the TF results (their position depends only upon $A_{\mu}$ ) and they should also disappear in the $I$ phase ${ }^{14}$ resonances with such properties are not observed. The $\Delta M=2$ transitions are usually invisible, so we infer that our resonances must all be $\Delta M=0$, one for each of the four possible $\mathrm{Mu}$ addition sites. 


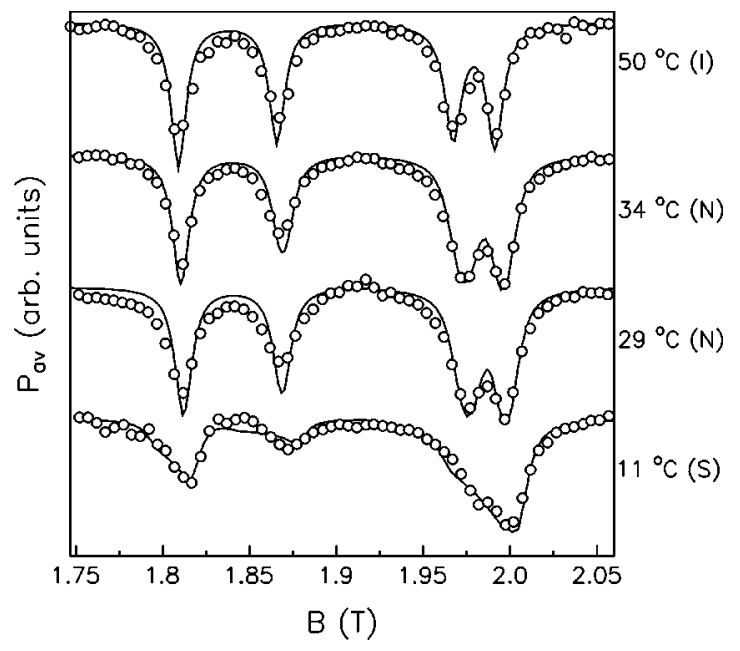

FIG. 4. ALC spectra taken from 5CB for four different temperatures.

For the I phase all dipolar parts of the Hamiltonian are averaged out, so we expect only the Fermi contact terms to contribute. The positions of the resonances then depend on $A_{\mu}$ and a proton-electron hyperfine constant $A_{n}$ [see Eq. (2.1)]. To first order the positions of the resonances $B_{r}$ are easily calculated by setting the level splitting to zero in the Paschen-Back high field regime:

$$
B_{r}=\frac{A_{\mu}-A_{n}}{2\left(\gamma_{\mu}-\gamma_{n}\right)} .
$$

The resonances have a model-dependent width, which varies with dynamical model and strength of dipolar coupling. ${ }^{14,15}$ We have already determined the four values of $A_{\mu}$ and so we use the high temperature ALC data to obtain values for $A_{n}$. We assume that the resonances appear in the same order as in the TF experiment and the consequent values of $A_{n}$ are shown in Table I. The fitted curves are shown in Fig. 4.

Our model for the $N$ phase contains three assumptions. First, the director is assumed to have the same symmetry as both the muon-electron and muon-nuclear dipolar coupling tensor since there is fast uniaxial rotation of the liquid crystal molecules around their long axis. Second, the director positions are assumed to be initially distributed uniformly around a cone of particular semiangle $\theta_{M} \cdot{ }^{16}$ Free energy arguments indicate that a small applied field aligns the molecules, but does not affect the expected range of angles around this mean (this is controlled by the free energy associated with the entropy of rod packing, which is much larger than that associated with the magnetic anisotropy). NMR and Raman measurements ${ }^{17}$ of the order parameter $P_{2}{ }^{1}$ in $5 \mathrm{CB}$ thus permit calculation of the cone semiangle at each temperature. Finally, fluctuations of the long molecular axis must be taken into account. These are controlled by thermal effects. We are mainly interested in those fluctuations which occur on the timescale to which this experiment is the most sensitive and in simulations a correlation time of $\tau_{c}^{\mathrm{av}}=1 \mu$ s gives the largest ALC signal for the $\Delta M=0$ transition. This is several orders of magnitude larger than the correlation times found in the previous section, indicative of the closer proximity of the energy levels in the ALC case. The correlation time $\tau_{c}$ of fluctuations depends on the wave number $q$,of the excitation according to ${ }^{1}$

$$
\tau_{c} \sim \eta / K q^{2}
$$

where $\eta$ is an effective viscosity which is similar in our case to the actual viscosity of $5 \mathrm{CB}^{1}$ and $K$ is a Frank elasticity constant. ${ }^{18}$ The one-Frank-constant approximation has been used here. The effective viscosity $\eta$ depends on the direction of the excitation through the liquid crystal, ${ }^{1}$ but we can use an orientationally averaged value in our simulations.

In order to obtain a higher cutoff wave number for important excitations we integrate this expression to a cutoff and obtain an expression for $\tau_{c}^{\mathrm{av}}$ :

$$
\tau_{c}^{\mathrm{av}}=3 \eta / K q_{c}^{2}
$$

Substituting our value of $\tau_{c}^{\mathrm{av}}=1 \mu$ s gives a minimum wavelength for excitation of about $400 \AA$, which is of the order of ten long molecular lengths. We therefore are justified in using a continuum model to extract the fluctuation angle of the long axis and so we can effectively identify the long axis fluctuations as director fluctuations. The angle $\beta$ around the initial orientation into which the director may roam can now be estimated by an application of the equipartition theorem, with the result that the mean square angle is ${ }^{1}$

$$
\left\langle\beta^{2}\right\rangle \approx k_{B} T q_{c} / \pi^{2} K
$$

where $q_{c}$ is an upper cutoff wave vector for fluctuations of the director about its mean position.

Combining Eqs. (4.4) and (4.3) we obtain the following expression for $\left\langle\beta^{2}\right\rangle$ which depends on a chosen $\tau_{c}^{\text {av }}, T$ and various constants:

$$
\left\langle\beta^{2}\right\rangle \approx \frac{k_{B} T}{\pi^{2}}\left(\frac{3 \eta}{K^{3} \tau_{c}^{\mathrm{av}}}\right)^{1 / 2} .
$$

We noted above that we use a correlation time $\tau_{c}^{\mathrm{av}}=1 \mu \mathrm{s}$ since this gives the largest ALC signal for the $\Delta M=0$ transition; in fact the absolute value of $\tau_{c}^{\text {av }}$ is not critical in determining the shape of resonances.

We also fix the electron-nuclear dipolar constant $D_{n}$ at 2 $\mathrm{MHz}$, a value typical of organic radicals ${ }^{14}$ and we assume that it is temperature independent to simplify the fitting procedure. Its effect is generally much smaller than the direct coupling to the electron. ${ }^{14}$ In the Monte Carlo simulation of $P_{\text {av }}$ the angle of each fluctuation is chosen according to an exponential probability distribution and reorientation happens with the correlation time $\tau_{c}^{\text {av }}$. We use the simulations to fit the data by varying only $D_{\mu}$ and the results are shown in Fig. 4 and in Table II. The fits are good and do not depend critically on the value chosen for $\beta$ and $\theta_{M}$ so the approximate assumptions used here do not detract from the validity of the results.

The $S$ phase results were fitted by making the simplifying assumption of static uniaxial couplings of proton and muon to the electron, ${ }^{5}$ which are also assumed to share a common axis. The orientations of the axis are assumed to cover all 
TABLE II. Fitted values of $D_{\mu}$ in the $N$ and $S$ phases. Columns $1,2,3$, and 4 refer to the resonance positions in ascending order of magnetic field. We estimate the error on each value to be $\pm 10 \%$.

\begin{tabular}{ccccc}
\hline \hline & \multicolumn{4}{c}{$D_{\mu}(\mathrm{MHz})$} \\
\cline { 2 - 5 }$T\left({ }^{\circ} \mathrm{C}\right)$ & 1 & 2 & 3 & 4 \\
\hline \multirow{2}{*}{11} & 4.5 & 3.6 & 7.2 & 4.8 \\
29 & 1.7 & 1.5 & 0.6 & 0.9 \\
34 & 1.7 & 1.3 & 0.7 & 1.0 \\
\hline \hline
\end{tabular}

angles with equal probability, which gives rise to a characteristically asymmetric line shape. Again, $D_{\mu}$ is the only free parameter for each resonance and the results are shown in Fig. 4 and Table II. The values are in reasonable agreement with those obtained for solid benzene in a zeolite cage. ${ }^{5}$

A comparison of the fitted values of $D_{\mu}$ displayed in Table II reveals a clear decrease for all four resonances on going from the $S$ phase into the $N$ phase. This is to be expected since there will be fast rotation of the molecules about their long axes. The amount of decrease is rather large (around a factor of 4) suggesting that in the $S$ phase the principal axis of the hyperfine tensor is at an angle of around $45^{\circ}$ from the long molecular axis.

We now see why the $\Delta M=1$ resonances are not seen at any temperature. In Ref. 14 it is shown that this resonance broadens significantly with faster dynamics and becomes weaker for a smaller $D_{\mu}$. This is in contrast to the $\Delta M=0$ resonances, which become narrower and have a larger amplitude for smaller $D_{\mu}$. This effect makes them much easier to observe in all phases in $5 \mathrm{CB}$, where $D_{\mu}$ is always relatively small.

\section{CONCLUSIONS AND FURTHER DISCUSSION}

We begin our discussion by comparing our results with those obtained by proton NMR. Many of the existing NMR data ${ }^{1}$ show sensitivity to dynamics on a similar timescale to that probed by ALC; the nuclear spin is mainly affected by fluctuations on a timescale comparable to the nuclear period $\omega_{k}^{-1}$, which is typically $0.1 \mu \mathrm{s}$. NMR detects dynamical effects through anisotropic proton-proton couplings and its specific advantage over $\mu$ SR is that the proton does not decay. In muon instruments the resolution is limited by the finite lifetime of the particle. This fact, however, has not prohibited us from studying the dynamics in $5 \mathrm{CB}$ since the muon forms a radical species and the unpaired electron produced couples much more strongly to the muon than do pro- tons with one another. Through the isotropic contact interaction of the electron we have been able to study smaller anisotropic perturbations which have revealed to us the molecular dynamical properties of 5CB.

In this study, we have also shown that muons have a large dynamic range when used in organic radicals; in the LF $\mu$ SR experiments the well separated energies of the four state $\mu^{+} e^{-}$system meant we were looking at fluctuations at the $10-100 \mathrm{ps}$ level, whereas the very close approach of states in the ALC measurement allowed us to probe dynamics at the microsecond level. This has allowed us to probe both the slower collective dynamical properties of $5 \mathrm{CB}$, which are due to the interactions between large numbers of molecules, and also the faster individual molecular motions.

Theories of NMR relaxation use principles similar to those we have used here; the most significant molecular motions are the small angle fluctuations of the director around its mean position. However, NMR results are somewhat more complicated, due in part to the effect of dipolar coupling of the aromatic protons of interest with the methyl protons at the ends of the aliphatic chain. Muons do not couple strongly to protons at such a large distance because the muon will naturally select sites of unsaturation and the radical spin is then located on the aromatic ring-precisely the parts of the molecule which give rise to mesogenic behavior. This to some extent circumvents the need for selective deuteration which is required for NMR studies in liquid crystals.

In conclusion, we have successfully detected and modeled the temperature dependent molecular dynamics in a nematic liquid crystal using the positive muon as a probe. Our work on bulk 5CB paves the way for a wider investigation of liquid crystal molecules in more constrained geometries, such as those found in the liquid crystal polymers. ${ }^{19}$ Muons have already been used to study other molecules in constraining environments, such as cyclohexadienyl radicals adsorbed onto the surfaces of silica grains ${ }^{20}$ and in silicalite zeolite cages. ${ }^{5}$ In a suitably chosen liquid crystal polymer, the muon would bind to unsaturated liquid crystalline parts of the polymer chain and avoid saturated linkages, thus providing a direct probe of the dynamics of mesogenic molecules.

\section{ACKNOWLEDGMENTS}

We wish to thank U. Zimmermann for valuable technical assistance and S. F. J. Cox, E. P. Raynes, and S. Elston for useful discussions. This work is supported by the EPSRC (U.K.).

\footnotetext{
*Email address: b.lovett1@ physics.ox.ac.uk

†Present address: Sektion Physik, Universität München, Theresienstraße 37, 80333 München, Germany.

${ }^{\ddagger}$ Also at RIKEN-RAL, Rutherford Appleton Laboratory, Chilton, Didcot OX11 0QX, U.K.

${ }^{1}$ P.G. de Gennes and J. Prost, The Physics of Liquid Crystals (Oxford University Press, New York, 1993).

${ }^{2}$ L. Onsager, Ann. N.Y. Acad. Sci. 51, 627 (1949).
}

\footnotetext{
${ }^{3}$ R.Y. Dong, Nuclear Magnetic Resonance of Liquid Crystals (Springer, New York, 1997).

${ }^{4}$ E. Roduner, The Positive Muon as a Probe in Free Radical Chemistry (Springer, Berlin, 1988).

${ }^{5}$ Muon Science, edited by S.L. Lee, S.H. Kilcoyne and R. Cywinski (IOP, Bristol, 1999).

${ }^{6}$ S.J. Blundell, Contemp. Phys. 40, 175 (1999).

${ }^{7}$ E. Roduner, K. Prassides, R.M. Macrae, T.M. Thomas, C. Nied-
} 
ermayer, U. Binninger, C. Bernhard, A. Hofer, and I.D. Reid, Chem. Phys. 192, 231 (1995).

${ }^{8}$ R.M. Macrae, K. Prassides, I.M. Thomas, E. Roduner, C. Niedermayer, U. Binninger, C. Bernhard, A. Hofer, and I.D. Reid, J. Phys. Chem. 98, 12133 (1994).

${ }^{9}$ S.F.J. Cox and D.S. Sivia, Appl. Magn. Reson. 12, 213 (1997).

${ }^{10}$ N. Bloembergen, E.M. Purcell, and R.V. Pound, Phys. Rev. 73, 679 (1948).

${ }^{11}$ C.P. Slichter, Principles of Magnetic Resonance (Harper and Row, New York, 1964).

${ }^{12}$ P.A. Beckmann, J.W. Emsley, G.R. Luckhurst, and D.L. Turner, Mol. Phys. 59, 97 (1986).

${ }^{13}$ J.S. Lewis, E. Tomchuk, H. Hutton, and E. Bock, J. Chem. Phys.
78, 632 (1983).

${ }^{14}$ S.R. Kreitzman and E. Roduner, Chem. Phys. 192, 189 (1995).

${ }^{15}$ P.L.W. Tregenna-Piggott, E. Roduner, and S. Santos, Chem. Phys. 203, 317 (1996).

${ }^{16}$ C.C. Wang and R. Pecora, J. Chem. Phys. 72, 5333 (1980).

${ }^{17}$ L.G.P. Dalmolen, S.J. Picken, A.F. de Jong, and W.H. de Jeu, J. Phys. (France) 46, 1443 (1985).

${ }^{18}$ M.J. Bradshaw, E.P. Raynes, J.D. Bunning, and T.E. Faber, J. Phys. (France) 46, 1513 (1985).

${ }^{19}$ S. Zumer and G. P. Crawford, Liquid Crystals in Complex Geometries (Taylor and Francis, London, 1996).

${ }^{20}$ I.D. Reid, T. Azuma, and E. Roduner, Nature (London) 345, 328 (1990). 